\title{
Segmentación \\ residencial e informalidad económica: un ejercicio de tipologías.
}

Residential segmentation and economic informality: an exercise in typologies.

Pablo Molina Derteano

Doctor en Ciencias Sociales

Titular de la asignatura Estudios Socio-

Demográficos

Carrera de Trabajo Social (FSOC-UBA)

pablomd2009@gmail.co

Fecha de recepción:

5.11.14

Fecha de aprobación:

14.6.15

\begin{abstract}
Resumen
El presente artículo es un estudio de caso de la configuración socio-territorial de un territorio segregado en la localidad de Ministro Rivadavia. Adoptando una perspectiva de estratificación, se muestra un anudamiento entre crecimiento de la informalidad económica y segregación residencial que perfilan espacios urbanos de gran complejidad en una localidad de reciente crecimiento demográfico. La metodología apunta a la construcción de tipologías, siendo que se considera como el mejor recurso para dar cuenta de la multidimensionalidad de las problemáticas que afectan a los hogares derivadas del anudamiento entre territorios y mercados de trabajo segmentados.
\end{abstract}

Palabras clave: Territorio, segregación, informalidad, tipologías, mercado de trabajo, Provincia de Buenos Aires.

\section{Abstract}

This article presents a case study of the socio-territorial configuration of a segregated area in Ministro Rivadavia, a township in Buenos Aires Province. Through an approach to social stratification, interactions between increases in economic informality and residential segregation lead to the emergence of urban areas of great complexity, in a locality of recent demographic growth. The methodol- 
Keywords: Territory, segmentation, informality, typologies, labor market, Buenos Aires Province.

\section{Introducción}

Los debates más recientes en torno los alcances de la recuperación económica de la última década y las políticas llevadas adelante por el Estado para la reducción de la pobreza y la indigencia reconocen que se debe prestar especial atención a los territorios urbanos. La evidencia empírica parece señalar que en determinados contextos territoriales - si bien se registran avances importantes - persisten obstáculos que dificultan la plena superación de condiciones de pobreza e indigencia (Clemente 2014). Más aún, se registran nichos de pobreza persistente en donde los riesgos de reproducción intergeneracional de las condiciones de segregación y pobreza se hacen presentes. Este artículo se propone describir el proceso de configuración de las áreas segregadas de Ministro Rivadavia, en el partido de Almirante Brown, ubicado en el segundo cordón del Gran Buenos Aires. El foco del análisis está puesto en un tema muy caro a la historia del Gran Buenos Aires, que es el anudamiento entre segregación residencial y economías duales. Tomando como estudio de caso la localidad de Ministro Rivadavia, se propone un análisis de tipologías para describir cómo se estructuran y articulan estos procesos.

\section{Coordenadas teóricas: la especificidad del enfoque territorial}

\subsection{Consideraciones generales.}

Las relaciones entre el concepto de territorio y las ciencias sociales han sido amplias, frondosas y tienen inclusive ciertos matices contradictorios (Rodriguez Vignoli 2001, Schneider y PeyréTartaruga 2006, Sosa Velazquez 2012, Echeverría 2014). Una revisión relativamente exhaustiva excedería el marco del presente artículo pero resulta necesario establecer algunos puntos de partida.

El primero es contar con una definición operacional sobre cuáles son los alcances del concepto de territorio. En este sentido, el territorio es considerado

no sólo como un espacio geográfico, sino principalmente como el resultado de la intervención de la sociedad sobre ese espacio. Esta noción del territorio como construcción social se despliega en varios sentidos: implica considerar de 
manera integral el abanico de dimensiones que confluyen en la constitución de los fenómenos socioespaciales, como también atender a los procesos dinámicos que generaron dichos fenómenos” (Roffman 2010:10).

De esta forma la mirada sobre una localidad del Gran Buenos Aires no dejará de considerar cierta historicidad de una tensión constitutiva de ese mismo espacio social (Echeverría 2014).

El otro punto de partida que se propone es la distinción entre territorio y enfoque territorial. Según Schneider y Peyré Tartaruga el territorio es un concepto heurístico que encierra tanto lo localización geográfica como el espacio social que más o menos delimita el primero. Pero un enfoque territorial, "presupone la acción sobre el espacio y el cambio de las relaciones sociales en él existentes" (Schneider y PeyréTartaruga 2006:85). En este sentido, este artículo se propone un enfoque territorial tratando de dar cuenta cómo se configuran relaciones sociales en su interior.

Ambos puntos de partida son bastante ambiciosos en principio y sus alcances lo suficientemente amplios para exceder el marco de este trabajo. Por ello, de esas modificaciones de las relaciones sociales, este artículo se ocupa únicamente de la segregación residencial socioeconómic.

Citando a Sabatini, Rodriguez Vignoli parte de una definición de la segregación espacial y residencial como "una aglomeración geográfica de familias de una misma condición o categoría social” (Rodriguez Vignoli 2001:12). Delimita así entre una tradición norteamericana que ha tendido a observar la segregación racial (o más bien, étnica), mientras que en América Latina la tradición de estudios se orientó a las diferencias socioeconómicas. Avanzando un poco más en la operacionalización, el autor señala que se trata de un grupo social que comparte características comunes y está localizado en una zona haciendo que la misma se vuelva homogénea en un espacio heterogéneo o potencialmente heterogéneo.

Otros autores, como Saraví $(2004,2008)$ comparten la idea de la segregación, aunque advierten que hay que distinguir entre diferenciación y segregación desde una perspectiva más micro. Los territorios urbanos son en primera instancia colindantes del fenómeno de diferenciación, sin necesariamente ser ésta de carácter segregante. Inclusive en contexto de pobreza, un barrio no necesariamente está segregado. Es decir, que para este autor la segregación no deviene necesariamente de diferencias objetivas, sino de formas de vinculación entre los grupos en el espacio social (ver también Barbosa 2001; Bourdieu 2002. Kessler (2014), por su parte, sostiene que para que el territorio tenga ese efecto de segregación, debe darse una combinación de ciertos rasgos de los hogares con 
falta de inversión en infraestructura. A su vez Sosa Velazquez (2012) llama la atención en la existencia de acuerdos previos en las relaciones de poder por las cuales un espacio geográfico pueda actuar como agente estigmatizante.

Puede decirse entonces que la segregación residencial tiene dos tipos de abordajes: 1) uno más directo que consiste en emplazar geográficamente uno o más grupos sociales que comparten rasgos objetivos que los puedan poner en una situación de desventaja, y 2) una corriente que supone la necesidad de estudiar si efectivamente se dan vínculos sociales y simbólicos que permitan hablar de segregación (la sola presencia de pobreza no bastaría), o inclusive falencias en infraestructura de servicios como escuelas o transporte (Palma Arce y Soldano 2010; Kessler 2014). Nuevamente, este contrapunto supera el espacio aquí disponible pero alcanza a poner reparos sobre la utilización del concepto de segregación, a menos de poder contar con un abordaje más antropológico o con ciertos indicadores de infraestructura de transporte entre otros. Por ello, se hablará de un efecto socioeconómico segmentante que viene del anudamiento entre la inserción en el mercado de trabajo y la residencia en una parte del barrio cuya infraestructura y condición de los hogares presentan rasgos precarios.

\subsection{Segmentación residencial y laboral. El rol de la heterogeneidad estructural.}

El enfoque territorial se detiene sobre el marco espacial de las relaciones dadas y las que pueden transformarse. Aquí interesa ver el marco de ciertas relaciones económicas y sociales entre los sectores formal e informal de la economía, interpelados fundamentalmente a través del concepto de heterogeneidad estructural. Ciertamente, hay una profusa bibliografía sobre las relaciones entre economía formal e informal, así como la dualidad de mercados de trabajo. ${ }^{1}$ Aquí interesa la forma en que esta dualidad tiene efectos sobre la estructura social y como esta estratificación podría tener un correlato en la conformación de un grupo -o más bien clases o fracciones de clase- que afectarían y estarían afectados por la segmentación espacial.

El enfoque de la heterogeneidad estructural -de acuerdo al economista chileno Anibal Pinto- enfatiza la conformación de economías duales de diferente productividad fruto del desarrollo dependiente de América Latina. Los bolsones de trabajadores informales urbanos -que Germani y otros autores identificaban con la marginalidad- eran resultantes de condicionamientos estructurales que daban como resultado un excedente relativo de mano de obra poco calificada que se empleaba -o mejor, se autoempleaba- en trabajos de servicios urbanos y pequeños talleres de baja productividad en condiciones precarias (Carpio y Novacoksky 2000; Quijano 2012).

De esta forma, desde el enfoque de la heterogeneidad estructural se afirma que en su propio funcionamiento la economía latinoamericana genera un sector de baja 
productividad y desprotegido. Autores como Tokman, en el marco del enfoque del PREALC-OIT, han enfatizado que la dualidad del mercado de trabajo produce efectos de estratificación, generando diferencias no sólo en las condiciones de vida, sino también de status (Tokman y De Souza 1978). Los mercados de trabajo urbanos -en este caso, del Gran Buenos Aires (GBA) - funcionaban de forma segmentada, limitando las ventajas del proceso de industrialización y de expansión de la economía moderna. Aun así, la expansión de servicios modernos como la salud, la educación y otros repercuten en una mejora de la calidad de vida si se la contrasta con entornos rurales.

Estos debates surgen en el marco de un proceso mediante el cual el conjunto de partidos circundantes a la Ciudad de Buenos Aires se expandieron desde la década de 1930, al ritmo de la industrialización por sustitución de importaciones. Fue un diagnóstico coincidente de la época que el proceso no era lo suficientemente dinámico para evitar la conformación de grandes bolsones de población cuyo rasgos no sólo abarcaban la pobreza sino la dificultad de beneficiarse con los aumentos de ingresos y derechos sociales y laborales iniciados con el primer peronismo (Echeverría 2014). A partir de la década de 1980, como consecuencia de la primera ola de reformas neoliberales impulsadas por el golpe de Estado de 1976, se fue consolidando un efecto de cierto aislamiento. El sector informal había funcionado hasta el momento como soporte del sector formal y como refugio cuando el ciclo económico empujaba a crisis de empleo. A partir de esa década, se fue fortaleciendo una economía informal que produce bienes y servicios para sectores de bajos ingresos y se va consolidando el rol de las microfirmas y de las economías de ferias como tomadores de mano de obra poco calificada y productores y distribuidores de bienes y servicios “para pobres” (Galin 2000; Chávez Molina 2010). Es decir, que el sector informal se va desenvolviendo con dinámicas propias, haciendo menos factible que quienes en él se desempeñen pueden pasar al sector formal (Molina Dertean, 2007; Lavopa, 2009), o inclusive, con esa inserción terminen por reproducir su situación misma de pobreza (Beccaria y Groissman 2008).

Tanto a nivel conceptual como empírico, la dualidad de economías formal e informal no solo tiene efecto sobre el ingreso y el status de los trabajadores y las trabajadoras, sino que además tiene un efecto sobre la estructura social, generando un proceso de segmentación y estratificación. Piore (1983), conocido por haber distinguido la dualidad de los mercados de trabajo, presenta un concepto accesorio y complementario que es el de cadenas de movilidad, el cual

representa un intento de formalizar la idea intuitiva de que el movimiento socioeconómico de nuestra sociedad no es aleatorio, sino que tiende a producirse a través de canales más o menos regulares. Estos canales son tales que un puesto de trabajo tenderá a ser cubierto por trabajado- 
res procedentes de un número limitado y característico de puntos concretos. Como consecuencia, la gente tiene empleos en un orden o secuencia regular. A esa secuencia la llamaremos cadena de movilidad (...) así pues la gente de un empleo dado tenderá a proceder de una limitada gama de escuelas, vecindades y tipos de características familiares; $y$, a la inversa, la gente que sale de la misma escuela o vecindad tenderá a entrar en una situación de empleo perteneciente a un conjunto limitado (Piore 1983:197-8).

Esta idea se relaciona con la metamorfosis antes mencionada por la cual el sector informal parece ser un boleto solo de ida para los trabajadores y trabajadoras de hogares pobres.

En este sentido, Piore vincula reproducción de la dualidad de mercados de trabajo con reproducción de desigualdades de origen, lo que lo aproxima, intuitivamente si se quiere, a un enfoque de estratificación social. Los estudios de Alejandro Portes, orientado al caso de los migrantes latinoamericanos en Estados Unidos, se convirtieron también en referencia en materia de sus análisis del sector informal, pero además Portes (1989) fue pionero en sugerir que la inserción en diferentes sectores de la economía da lugar a la conformación de distintas clases sociales. En un sentido similar, Chávez Molina y Sacco (2014) presentan un esquema de clases denominado Clasificador Ocupacional Basado en la Heterogeneidad Estructural (COBHE), en el que se pone como ejes del proceso de estratificación la calificación y la productividad de la economía, distinguiendo a asalariados, cuentapropistas y empleadores según su inserción en el sector formal o informal (Chávez Molina y Sacco 2013).

Estos aportes son pinceladas de una cuestión más amplia como es el de la estructura social, pero contribuyen a dar cuenta de que la heterogeneidad estructural no sólo genera una segmentación de mercados de trabajo o de sectores productivos, sino que además la produce en la estructura social. Si la heterogeneidad estructural, como lo sugieren directamente Chávez Molina y Sacco e indirectamente Portes, tiene efectos de estratificación y la vivienda y el barrio son indicadores, entre otros, de la posición en la estructura de clases, sería muy pertinente interrogarse en qué medida la inserción socio ocupacional y la segmentación residencial están relacionadas, pero además cómo pueden condicionar otros rasgos de los hogares.

Sobre este punto, conviene presentar las hipótesis de trabajo que irán guiando la indagación. Estas hipótesis se preguntan por el anudamiento entre: 1) un efecto de segmentación territorial tal que los hogares ubicados en un mismo espacio social geográficamente delimitado tengan rasgos homogéneos en términos socioeconómicos, y 2) un efecto 
de segmentación de la condiciones socioeconómicas de los hogares resultante de la participación en el sector informal por parte del principal sostén económico de los hogares (PSH). Este anudamiento resultaría en la conformación de perfiles de hogares de acuerdo a la inserción socio ocupacional del PSH y la localización dentro del barrio. En otras palabras, ¿pueden la posición ocupacional y la localización geográfica actuando en conjunto estructurar los rasgos de los hogares concentrando sus rasgos vulnerables y no vulnerables? De un modo muy simplificado, se podría esperar que todos los hogares no vulnerables y cuyos jefes tengan empleos registrados con cobertura social y previsional se ubicaran en una misma área geográfica mientras que todos los vulnerables con jefes y jefas con empleo no registrados se ubicaran en otro. Una distribución de esta naturaleza daría cuenta de la capacidad altamente discriminante de ambas dimensiones -lo territorial y el mercado de trabajo-, en la conformación de, al menos, dos tipos de hogares, vulnerables y no vulnerables. Pero como se verá más adelante, el análisis mostrará que hay más de dos tipos posibles.

\section{Datos y metodología}

\subsection{Datos y estudio de caso}

Se procede a continuación a describir los resultados de un estudio realizado en 2008 en la localidad de Ministro Rivadavia. La metodología es cuantitativa y los análisis son de carácter descriptivo; utilizando como fuentes de datos secundarios los Censos Nacionales de Población y Vivienda de 1991, 2001 y 2010; relevamientos de ACUMAR (Autoridad de la Cuenca Matanza-Riachuelo) y del propio municipio de Almirante Brown. ${ }^{2}$ La encuesta de hogares indagó por las características de los mismos y se propuso contribuir a la construcción de datos para describir: a) las características habitacionales de los hogares en términos de la infraestructura del barrio y de la vivienda propiamente dicha; b) la composición del hogar en términos de PSH activo y miembros inactivos y/o desempleados.

Metodológicamente, es preciso señalar que se trata de un estudio de caso. Hay un acuerdo en definir al estudio de caso como una observación sostenida y en profundidad de uno o muy pocos casos (Flyvberg 2004, Arzaluz Solano 2005:). Por su parte, Yin (1994) señala que el estudio de caso no es en sí mismo una técnica, sino una forma de organizar los datos; ya que resulta imposible - sobre todo en sociología - dar cuenta de lo peculiar de una unidad de estudio sin utilizar conceptos y herramientas más generales. Si bien hubo en la tradición sociológica más temprana muchas indagaciones del tipo del estudio de caso, hubo desde la escuela de Chicago una marcada tendencia a inscribirlos territorialmente: regiones, barrios, guetos, etc. (Arzaluz Solano 2005.

La primera advertencia metodológica que suele hacerse cuando se trata de estudios de caso es que no es posible generalizar, en el sentido de establecer descripciones o 
explicaciones causales que -en caso de verificarse positivamente- puedan ser extrapoladas y declaradas válidas para otras unidades. El estudio de caso es antitético al diseño experimental y la comparabilidad (Yin 1994,Arzaluz Solano 2005), así como la generalización de tipo estadística. Sin embargo, esto no quiere decir que no haya otras formas de validación y, en este sentido, el presente es un estudio de caso crítico que propone elementos para la construcción de hipótesis susceptibles de ser testeadas y comparadas en unidades más grandes y diversas.

\subsection{La tipología como perspectiva y herramienta de análisis}

El análisis aquí propuesto responde a una metodología de construcción de tipologías. La construcción de tipologías como método de análisis en Ciencias Sociales tiene una rica tradición y quizás tenga en la figura de Max Weber a uno de sus máximos referentes. Weber distingue entre tipos ideales ${ }^{3}$ y tipos construidos, siendo estos últimos los que emplearemos aquí. Respecto al tipo construido, McKinley sostiene que "puede prestar el importante servicio de funcionar como puente entre la teoría sistemática sustantiva y los datos empíricos relativamente no estructurados" (en Gomez Rojas y Riveiro, 2014:91). Los autores también citan a Allan Bartón, una referencia en esta temática, que vincula al concepto de tipología con el espacio de propiedades, según la conocida formulación de Lazarsfeld. Como todo proceso de operacionalización, la tipología es una herramienta tanto teórica como empírica, que parte de tres operaciones: ordenamiento, sistematización y resumen de indicadores en una misma herramienta. Una tipología tiene -o debería tener- su mayor fortaleza en ser homogénea en su interior y heterogénea al exterior. El conjunto de hogares que cayeran dentro de una misma tipología debería tener rasgos muy similares entre sí, mientras que esos mismos rasgos los distinguen de otros tipos.

Las técnicas empleadas para construir tipologías van desde procesos relativamente simples como formas de ponderar índices hasta combinatorias entre análisis de conglomerados y de correspondencias múltiples. Si bien se ha elegido este último, es importante detenernos sobre cierta controversia existente en torno a la construcción y validación. Para algunos investigadores resulta primordial la robustez teórica de las tipologías, mientras que para otros, es muy importante la validación empírica apoyándose en el poder de los softwares actuales (SPSS, SPAD, etc). Ambas posiciones, pueden lamentablemente conducir a cierto reduccionismo. La primera asemejaría más a la construcción de tipos ideales antes que tipos construidos en donde el hallazgo sería tratar de reducir la "desviación empírica" con respecto a los conceptos teóricos. La segunda consiste en apoyarse en los agrupamientos de categorías y/o indicadores que puedan realizarse en el plano factorial abandonando a la validación estadística todo rol de construcción.

Aquí se elige una posición intermedia, ya que los ejes claves de construcción devienen 
de las observaciones antes realizadas sobre heterogeneidad estructural y análisis territorial. Lo que articula a ambas coordenadas de análisis es que se parte de que ambas tienen un efecto segmentador.

La metodología y el tipo de análisis que se utilizan siguen líneas muy similares a las propuestas por López Roldán (1996; Ardiles y Molina, 2013). Aunque enfocado al mercado de trabajo, sus estudios proponen el análisis de las tipologías como herramientas para dar cuenta de una estructuración de la segmentación. Para López Roldán se trata de un tipo de investigación atributiva, que no sólo identifica y describe tipos, sino que les atribuye determinadas características. En este sentido, señala que se trata de una tipología que estructura y articula. En este punto, la técnica de análisis permite ver cómo se agrupan las categorías de las variables en un número determinado de perfiles. Se trata de un tipo de análisis multidimensional, que busca medir las distancias existentes entre los diferentes atributos y ubicar en qué plano del espacio social se acercan más. Para ello combina una técnica de agrupamiento en base a clusters con la selección de las asociaciones más robustas a través del análisis de correspondencias múltiples (ACM).

\section{El análisis}

\subsection{Contextualización: crecimiento poblacional}

Tradicionalmente, los partidos circundantes a la Ciudad Autónoma de Buenos Aires (CABA) han sido objeto de estudio y tomados como proxy de la estructura social argentina debido a su alto peso poblacional con respecto al resto del país, a la concentración de la población y al proceso de industrialización sustitutiva de importaciones que se inició en la década de 1930, así como fuera también el escenario de las grandes movilizaciones del peronismo. En 1947, la población de los partidos del GBA se ubicaba por debajo de la Ciudad de Buenos Aires o del resto de la provincia; pero a partir de entonces habría de tener lugar un proceso de crecimiento constante. Hacia fines de la década de 1950 el GBA superaba en población a la Ciudad de Buenos Aires y al resto de la provincia (Echeverria, 2014). Este crecimiento estaba principalmente motorizado por la industrialización, pero inclusive después de que este proceso fuera interrumpido, la población del GBA continuó creciendo y los otros dos distritos siguieron amesetándose y creciendo muy lentamente. Para el 2010, los 24 partidos del GBA tenían 3,43 veces la población de CABA y 1,73 veces la población del resto de la provincia que incluye aglomerados densamente poblados como La Plata y Mar del Plata. O sea que en primer término, se tiene que se trata de un espacio de 24 partidos que está densamente poblado y cuya tendencia de crecimiento poblacional no ha cambiado.

Siguiendo las coordenadas propuestas por la Dirección Provincial de Estadística de la Provincia de Buenos Aires (en adelante:DPEBA), el partido de Almirante Brown se ubica en la zona denominada Periurbana Sur -que abarca los partidos de Almirante Brown, 
Berazategui, Esteban Echeverría, Ezeiza y Florencio Varela-; se trata de una zona que ha tenido un crecimiento importante. Teniendo en cuenta la dinámica de crecimiento poblacional antes descrita, puede observarse cómo esta zona periurbana ha tenido una evolución que sigue las líneas generales pero que presenta particularidades.

- En términos horizontales y de acuerdo a fuentes censales, en el período intercensal 1991-2001 ha crecido un 23,5\%, siendo la segunda área de crecimiento detrás del periurbano oeste ${ }^{4}$ para el período 2001-2010, la zona periurbana sur creció un $16,7 \%$, quedando esta vez tercera en variación ascendente al ubicarse detrás del periurbano oeste y del conurbano oeste. ${ }^{5}$ Más allá de las posiciones, queda claro que es una de las zonas cuyo crecimiento poblacional es de los más pronunciados; ${ }^{6}$

- Al compararse con las zonas que componen la provincia de Buenos Aires, también se puede notar una tendencia de crecimiento diferencial considerable. En 1947 la población de las zonas que componen los 24 partidos, representaba $40,5 \%$ de la población de la provincia; en 2001, el 62,9\% y en 2010, el 63,4\%. En el marco de este crecimiento, puede observarse el marcado incremento de la zona periurbana sur: en 1947, representaba el 1,6\% de la población de la provincia; en 2001, alcanzó el 11\% y en 2010 el 11,3\%. En una mirada más global, son el conurbano oeste -motorizado sobretodo por La Matanza- y el periurbano sur, las dos áreas que experimentan un mayor crecimiento en su peso poblacional sobre la provincia entre 1947 y 2010. En cambio, las dos zonas mayoritarias que son el conurbano sur $^{7}$ y conurbano norte ${ }^{8}$ experimentan reducción de su peso poblacional. ${ }^{9}$

Tabla 1: Peso poblacional de las diferentes áreas, Provincia de Buenos Aires 1947-2010

\begin{tabular}{|l|l|l|l|l|l|l|l|}
\hline \multirow{2}{*}{ Área } & \multicolumn{7}{|c|}{ Censo } \\
\cline { 2 - 8 } & $\mathbf{1 9 4 7}$ & $\mathbf{1 9 6 0}$ & $\mathbf{1 9 7 0}$ & $\mathbf{1 9 8 0}$ & $\mathbf{1 9 9 1}$ & $\mathbf{2 0 0 1}$ & $\mathbf{2 0 1 0}$ \\
\hline Periurbano Norte & 3,9 & 6,9 & 8,4 & 10,3 & 10,8 & 11,3 & 11,2 \\
\hline Conurbano Oeste & 3,1 & 8,3 & 11 & 13,2 & 14,3 & 15,2 & 17,6 \\
\hline Periurbano Sur & 1,6 & 4,7 & 6,6 & 8,2 & 9,7 & 11 & 11,3 \\
\hline Conurbano Sur & 18 & 18,1 & 17,7 & 16,2 & 15,1 & 13,7 & 12,8 \\
\hline Conurbano Norte & 13,9 & 17,8 & 17,6 & 15,1 & 13,3 & 11,7 & 10,5 \\
\hline $\begin{array}{l}\text { Resto de la } \\
\text { provincia }\end{array}$ & 59,5 & 44,2 & 38,7 & 37 & 36,8 & 37,1 & 36,6 \\
\hline
\end{tabular}

Fuente: Elaboración propia a partir de datos de la DPEBA 
Es importante destacar que se trata entonces de una zona cuya población general ha experimentado un proceso importante de expansión. El partido de Almirante Brown se ubica dentro de esta tendencia de crecimiento reciente, pero el proceso fue relativamente más acelerado para el caso de Ministro Rivadavia.

Los datos de Ministro Rivadavia se muestran relativamente dispares de los del municipio. En parte, porque se trata de una localidad periurbana y mucho menos densamente poblada que el resto del partido. En este sentido, resulta interesante hacer algunas comparaciones entre el nivel partido y el nivel localidad.

Según los datos censales de 1991, vivían en Ministro Rivadavia 9.560 personas, y para 2001 se incrementaron a 16.535; lo que indicaba un crecimiento de 72\%. En el censo de 2010, se observa un crecimiento muy leve pasando a 16.740 habitantes (un aumento de 1\%). En el mismo período intercensal el municipio creció 14,4\% y luego 7,1\%. Es decir, que la demografia de la localidad se mueve de manera diferente del total del municipio dando lugar a su particularidad (AA.VV., 2010; INDEC, 2011; ACUMAR, 2013).

En este punto, es interesante destacar que el proceso de segmentación que se buscará analizar más adelante se hace con cierta sedimentación. En un contexto en donde el periurbano sur aumentó mucho su peso poblacional en la provincia y en donde el partido de Almirante Brown siguió esta línea, el crecimiento de Ministro Rivadavia fue casi nulo. Es de esperar que las dinámicas territoriales se tensen con nuevas urbanizaciones u ocupamiento de tierras o cambios en la estructura habitacional como resultado de un crecimiento tan pronunciado. En el caso de Ministro Rivadavia, el volumen de la población no ha aumentado significativamente si bien los datos de un relevamiento sugieren mayor presencia de niños y adolescentes. En este sentido, debe tenerse en cuenta que, más allá de lo que pueda verse en las tipologías, es posible inferir que se trata de un proceso de continuidad o reacomodamiento, el cual excede este artículo.

Finalmente, para poder avanzar con nuestro análisis, se intentará trazar una división socio-territorial del barrio en dos grandes zonas. Dichas zonas tienen la particularidad de ser estadísticamente significativas. Es decir, cuando ciertos indicadores de NBI son considerados en base a esta división se puede encontrar diferencias importantes. La población de mayores recursos tiende a concentrarse en las arterias principales que son las avenidas 25 de Mayo y Espora; a estas arterias las atraviesa una avenida importante que es la Avenida República Argentina. La llamada zona alta se ubica a la izquierda del mapa a continuación en dirección a las avenidas Espora y 25 de Mayo; puede verse que se trata de un espacio mucho más urbanizado. La que se denomina zona baja se encuentra hacia la derecha, por debajo de la diagonal que traza esta última avenida y también se puede apreciar que es una zona con menor urbanización. 
Figura 1: Mapa de la zona de relevamiento.

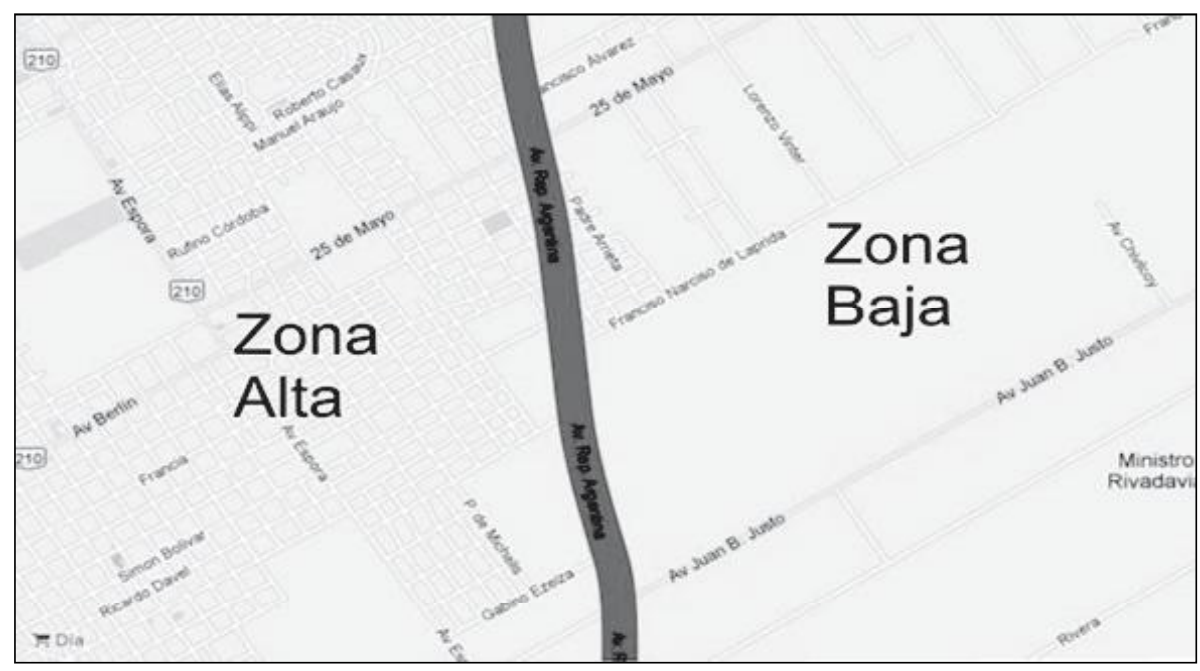

Fuente: Elaboración propia sobre Google Maps

\subsection{Análisis de la segmentación}

El objetivo del análisis que se describe a continuación es mostrar la dimensión segmentadora y estructurante de la localización barrial. Por segmentadora se entiende que las categorías consideradas más desfavorables de la localización barrial (zona baja reciente y residente) tenderían a coincidir con las inserciones ocupacional de peor calidad de los y las jefes/as. Esta asociación sería segmentadora del espacio social y laboral. Por estructuradora (y articuladora), se entiende que esta asociación condiciona otras dimensiones de la vida de los hogares así como rasgos demográficos y educativos de los jefes y jefas de hogar.

López Roldán et al (1996) señalan que toda operación dentro de un diseño metodológico se trata de una articulación entre supuestos teóricos y su validación metodológica. En este sentido, el primer paso fue determinar qué variables servirían de dimensiones clave del análisis. Estas dimensiones serían las "que tracen" el plano en donde se inscribirían y asociarían las otras variables y categorías. Por interés teórico, se determinó que una de estas variables fuera la ubicación de la vivienda de referencia que es lo que permite ver la localización barrial. ${ }^{10} \mathrm{Y}$ coincidente con la hipótesis la que tiene mayor inercia fue inserción socio-laboral.

En primer lugar, en la Tabla 2 se puede ver la contribución de cada una de las categorías de inserción ocupacional en la localización barrial. Se aprecia que la mayor contribución a la zona alta corresponde a los empleadores, independientes y asalariados formales, lo que está de acuerdo a los lineamientos antes descritos. La contribución de los trabaja- 
dores protegidos y/o integrados a la esfera formal de la economía alcanza poco más de las tres cuartas partes de quienes residen en la zona alta desde más de 10 años, y a más de dos tercios de los residentes recientes en esa zona.

La contribución a la zona baja reciente es considerable por parte de los trabajadores asalariados informales y de changarines, trabajo en hogares y beneficiarios de planes sociales. La categoría de empleadores informales, sin embargo, se muestra más heterogénea en su participación (Tabla 2)

Este ejercicio sirve como primera aproximación de carácter descriptivo al mostrar la asociación y sentido de la misma entre ambas variables. Se mostró asimismo que hay una importante contribución de las categorías de la variable inserción ocupacional a la distribución de las categorías de localización barrial, y que la asociación entre ambas variables es lo suficientemente fuerte para llevar a un análisis de correspondencia múltiple. ${ }^{11}$

Tabla 2: Contribución de la variables inserción ocupacional a las categorías de localización barrial.

\begin{tabular}{|c|c|c|c|c|c|c|}
\hline \multirow[b]{2}{*}{$\begin{array}{l}\text { Localización } \\
\text { barrial }\end{array}$} & \multicolumn{6}{|c|}{ Inserción ocupacional } \\
\hline & $\begin{array}{l}\text { Empleadores e } \\
\text { independientes }\end{array}$ & $\begin{array}{l}\text { Asalariados } \\
\text { formales }\end{array}$ & $\begin{array}{l}\text { Empleadores } \\
\text { informales }\end{array}$ & $\begin{array}{l}\text { Asalariados } \\
\text { informales }\end{array}$ & $\begin{array}{l}\text { Changas y } \\
\text { trabajo en } \\
\text { hogares }\end{array}$ & Total \\
\hline $\begin{array}{l}\text { Zona alta } \\
\text { residente }\end{array}$ & 0,45 & 0,31 & 0,13 & 0,11 & 0 & 1 \\
\hline $\begin{array}{l}\text { Zona alta } \\
\text { reciente }\end{array}$ & 0,33 & 0,36 & 0,19 & 0,10 & 0,02 & 1 \\
\hline $\begin{array}{l}\text { Zona baja } \\
\text { residente }\end{array}$ & 0,06 & 0,22 & 0,27 & 0,34 & 0,15 & 1 \\
\hline $\begin{array}{l}\text { Zona baja } \\
\text { reciente }\end{array}$ & 0 & 0 & 0,18 & 0,39 & 0,43 & 1 \\
\hline
\end{tabular}

Fuente: elaboración propia

\section{Las tipologías resultantes}

La acción estructuradora y articuladora sobre el plano social que tendrían la situación ocupacional y habitacional tiene un efecto sobre las categorías de otras variables que también son consideradas parte del análisis. En este sentido, la agrupación de las categorías dio lugar a tres tipos. Cada tipo fue identificado por la proximidad entre categorías de las variables eje y otras analíticas.

El gráfico que se presenta a continuación resume las coordenadas que van de menor a mayor con las dos variables elegidas. A los fines de hacer más comprensible la des- 
Pablo Molina Derteano

cripción se han eliminado las categorías sin asociación así como las variables que no presentan proximidad a alguno de los tipos.

Gráfico 1: Tipología formada con las coordenadas de localización barrial e inserción ocupacional + variables analíticas seleccionadas.

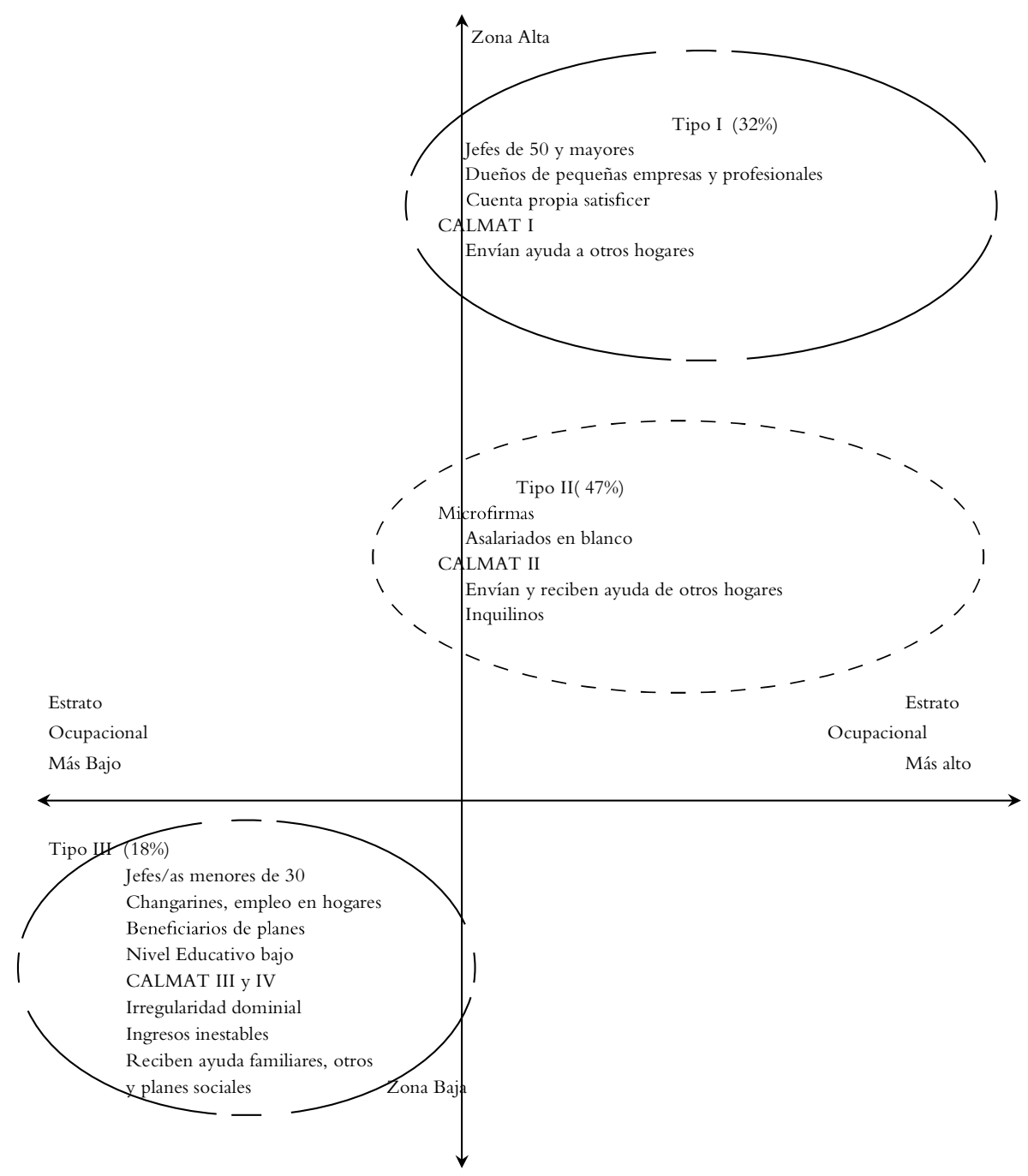

Fuente: Elaboración propia 


\section{Cuadro 1: Tipo I: Emprendedor y protegidos}

\begin{tabular}{|l|l|}
\hline Variable & $\begin{array}{l}\text { Categoría/s que se presentan en su totalidad o } \\
\text { en un porcentaje superior al } \mathbf{8 0} \%\end{array}$ \\
\hline Localización barrial & Zona alta reciente y residente \\
\hline Situación ocupacional & Empleadores y cuentapropia independientes \\
\hline Calidad de la vivienda & Optima - CALMAT I \\
\hline Propiedad de la vivienda & Tenencia Regular \\
\hline Valoración de los ingresos & Estables \\
\hline Percepción de programas sociales & No \\
\hline Edad (del jefe/a) & 50 y más \\
\hline Reciben o envían ayuda & Envían ayuda \\
\hline
\end{tabular}

Fuente elaboración propia

El presente tipo se compone por los hogares considerados como de mejor situación dentro del barrio. Se trata de residentes antiguos y resientes en la zona alta y sus viviendas se describen como suficientes teniendo inclusive detalles de terminación. Se la caracteriza como escala CALMAT I que se define como una vivienda que presenta materiales resistentes y sólidos en todos los parámetros (pisos, paredes o techos) e incorpora todos los elementos de aislación y terminación..$^{12}$ La zona alta no presenta falencias en la recolección de residuos o iluminación ni tampoco en el pavimentado de las calles. Tampoco son zonas inundables o de basurales.

Respecto a su situación ocupacional, está compuesto mayormente por propietarios de pequeños comercios o talleres y en menor medida por profesionales independientes. Éstos últimos son los profesionales liberales tradicionales (médicos, abogados, etc.) y profesionales en función específica en unidades productivas de envergadura. Este grupo fue un componente especialmente importante de la clase media argentina durante los años de la sustitución de importaciones. Inclusive se trata del máximo escalafón de ascenso de las clases medias que protagonizaron el pasaje de barrio obrero a barrio integral.

Pero no todos los independientes son profesionales liberales, sino que también hay trabajadores por cuenta propia en actividades vinculadas a la producción de bienes y servicios. Componen el grupo que fuera conocido como "cuentapropiasatisficer" (Beccaria, 1996). Se trata de trabajadores especializados con grados técnicos u operativos pero que trabajan por su cuenta. Estos trabajadores fueron un signo distintivo del desarrollo de los centros urbanos del Gran Buenos Aires, Gran Rosario y Gran Córdoba, entre otros. Una parte se volvió propietario de pequeños talleres de herrería, cerrajería, carpintería 
y otras especialidades que abastecían a las industrias cercanas. Sus dueños, muchas veces habían sido empleados en esas mismas fábricas; y estos micro talleres también tenían su clientela en el barrio prestando ese mismo servicio a particulares (Beccaria, 1978; Molina Derteano 2014). Otros prestaban directamente servicios especializados a particulares como arreglos de casas, electrodomésticos y otras actividades no manuales. Sus ingresos así como su status eran valorados socialmente como pertenecientes a la clase media, apoyándose en el imaginario del autoempleo: "no tenés jefes ni horarios" (Carpio et al. 2000).

Otro grupo lo constituyen los empleadores con negocios cuya actividad se encuentra en las ramas de comercio y/o servicios. Cuentan con pocos empleados, aunque su actividad está registrada, al igual que la de los "cuentapropiasatificer"

Respecto a sus estrategias de hogar, se destaca que en este tipo caben aquellos que señalan ayudar a otros hogares de familiares. Un rasgo demográfico interesante a destacar es que aquí se ubican los/las jefes/jefas de hogar mayores de 50 años, probablemente como resultado del proceso de acumulación en sus trayectorias individuales iniciadas previamente a la dictadura 1976-83.

\section{Cuadro 2: Tipo II: de baja vulnerabilidad}

\begin{tabular}{|l|l|}
\hline Variable & $\begin{array}{l}\text { Categoría/s que se presentan en su totalidad o en } \\
\text { un porcentaje superior al } \mathbf{8 0} \%\end{array}$ \\
\hline Localización barrial & Zona alta reciente y residente \\
\hline Categoría ocupacional & $\begin{array}{l}\text { Asalariado en blanco } \\
\text { Empleadores informales, dueños de microfirmas }\end{array}$ \\
\hline Calidad de la vivienda & Suficiente- CALMAT II \\
\hline Propiedad de la vivienda & Tenencia regular o inquilinato \\
\hline Valoración de los ingresos & Estables \\
\hline Percepción de programas sociales & No \\
\hline Reciben o envían ayuda & Reciben y envían a familiares \\
\hline
\end{tabular}

Fuente elaboración propia

También ubicados dentro de la ya descrita zona alta, se trata de residentes antiguos y recientes cuyas viviendas se describen como suficientes; aunque algunas, según los datos recabados, exhiben la falta de detalles de terminación. Componen lo que se denomina CALMAT II, que son viviendas con materiales resistentes y sólidos en todos los parámetros, aunque le faltan al menos un elemento de terminación o aislación en algún parámetro. Lo que resulta interesante es que los hogares que integran este tipo son propietarios pero también hay un importante número de inquilinos entre sus filas. 
Respecto a la situación socio-ocupacional, destaca la presencia de empleadores informales que poseen una serie de comercios tales como kioscos, almacenes, librerías con artículos escolares, carnicerías, etc. También hay pequeños talleres con ocupaciones manuales. Se trata del grupo que Galín (2000) denominara microfirmas para referirse a estos pequeños locales que se caracterizan por una escasa inversión de capital, relativamente poca división de las tareas y vínculos de tipo informal muchas veces basados en lazos fuertes (Granovetter, en Pérez, 2006). Se caracterizan como unidades productivas que están en más de un sentido, ancladas en lo local por su baja productividad y cobertura (Tokman, 2002). Funcionan como soporte de la economía formal, proveedoras de insumos a bajo costo (como el caso de los talleres textiles) o inclusive como proveedoras de bienes y servicios de muy bajo costo para los propios asalariados precarios (Chavez Molina 2014).

El otro grupo que aparece ligado son los asalariados del sector público y privado, en tareas manuales y no manuales pero con el rasgo de contar con empleo registrado. Forman un grupo ocupacional de trabajadores que requieren de algún grado de calificación y tienen alguna autoridad, pero escaso control del proceso productivo como por ejemplo los supervisores. Debe destacarse que estos puestos requieren en general de algún grado de calificación al que se adquiere con capital educativo.

Finalmente, respecto a sus estrategias este tipo se distingue porque no reciben ayuda de planes sociales y califican sus ingresos como regulares. Tienen vínculos de reciprocidad económica con familiares ya que brindan y reciben ayuda por parte de los mismos.

\section{Cuadro 3: Tipo III: de alta vulnerabilidad}

\begin{tabular}{|l|l|}
\hline Variable & $\begin{array}{l}\text { Categoría/s que se presentan en su totalidad o } \\
\text { en un porcentaje superior al } \mathbf{8 0} \%\end{array}$ \\
\hline Localización barrial & Zona baja reciente y residente \\
\hline Situación ocupacional & $\begin{array}{l}\text { Asalariado informal } \\
\text { Changas, trabajo en hogares y planes sociales }\end{array}$ \\
\hline Calidad de la vivienda & Precaria- CALMAT III y IV \\
\hline Propiedad de la vivienda & Tenencia irregular \\
\hline Valoración de los ingresos & Inestables \\
\hline Percepción de programas sociales & Si \\
\hline Edad (del jefe/a) & Hasta 29 años \\
\hline Reciben o envían ayuda & $\begin{array}{l}\text { Otros (terceros) } \\
\text { Familiares }\end{array}$ \\
\hline
\end{tabular}

Fuente elaboración propia 
El presente tipo se compone de los hogares considerados más vulnerables ya que todos residen en la zona baja y en su mayoría se trata de trabajos sin protección y muchas veces inestables. En este tipo se encuentran todos los hogares con jefatura de menores de 30 años.

En cuanto a la calidad de la vivienda, también aquí se concentran la totalidad de casos en que presentan viviendas inadecuadas o precarias (casillas, piezas o vivienda en terreno ajeno, otros), y casi la mitad de quienes componen esta tipología. Las edificaciones se encuentran principalmente dentro del valor III de la escala CALMAT, lo que quiere decir que se trata de viviendas sólidas y resistentes pero sin detalles de terminación o aislamiento. Otras, sobre todo las de un asentamiento, se ubican en el valor IV siendo viviendas que no son consideradas sólidas o resistentes. También se destaca que poco más de un tercio de las viviendas se ubica cerca de basurales y/o zonas inundables o con deficiencias en la iluminación o el retirado de residuos. Completa el panorama que casi la totalidad de los hogares con régimen de tenencia irregular se concentran en este tipo. En resumen, son viviendas precarias, en zonas desfavorables y con problemas de regularidad dominial. También se encuentran dentro de este grupo la mayoría de los jefes/jefas jóvenes.

En una descripción cualitativa se puede encontrar que este tipo se compone de trabajadores en changas u ocupaciones irregulares; empleo en hogares y asistidos por planes sociales. El primer grupo refiere a trabajadores manuales no calificados, muchos de los cuales se desempeñan en tareas eventuales como changas, principalmente de construcción. La cohesión interna y el grado de regularidad de sus prácticas son mucho más bajos que los estratos anteriores. Se trata de ocupaciones de baja o nula productividad con rasgos de autoempleo que sirven para garantizar un sustento diario. Otros en cambio son asalariados de pequeños establecimientos informales en condiciones de gran precariedad.

El segundo grupo refiere al empleo doméstico. Las trabajadoras domésticas inclusive representaban una estrategia de los hogares en tiempos de profundas crisis y constituyen una oferta relativamente estable en el tiempo, aún con la introducción de programas de transferencia de ingresos.

Finalmente queda un grupo, conformado por los trabajadores asistidos por programas de transferencia de ingresos. Éstos han surgido en los últimos años por los cambios de las políticas sociales. En algunos casos, estos beneficiarios deben realizar alguna contraprestación por pocas horas semanales (menos de 20), pero en el caso del Plan Manos a la Obra recibían el estímulo para comenzar emprendimientos productivos (EP). Según los datos de la evaluación de medio término, en la zona del GBA predominaron los emprendimientos textiles y de producción de alimentos (SIEMPRO, 2007:69). De hecho 
en el barrio, los arreglos de costuras y otros emprendimientos marginales relacionados a lo textil ocuparon un lugar destacado junto con la fábrica artesanal de ladrillos y otras ocupaciones artesanales

Más allá de este tipo de iniciativas, hay una marginalidad periurbana de gran tradición en el barrio y una actividad frecuente es el cultivo de micro-huertas para su venta ambulante. Otras actividades incluyen la prestación de servicios personales. Éstas y otras actividades componen un grupo ocupacional que puede definirse como cuentapropia de subsistencia en la medida que son autoempleados pero carecen de los recursos o la planificación para poder llevar adelante emprendimientos que no superen la reproducción diaria (Chávez Molina, 2011)

Con respecto a las estrategias de vida, prevalece una caracterización de sus ingresos como inestables, lo que se relaciona con el tipo de ocupaciones predominantes. A su vez, se destaca que reciben ayuda de familiares y terceros incluyendo iglesias, ONG, comedores, etc. además de que en algunos hogares se perciben ingresos por vía de planes sociales como el Plan Familias o el Plan Vida. Hay, como ya se mencionó, una importante parte de los hogares cuyos ingresos provenían en principio del plan Jefes y Jefas y luego fueron reemplazados por la Asignación Universal por Hijo o por emprendimientos productivos bajo la órbita del Plan Argentina Trabaja.

\section{Observaciones finales}

Retomando la hipótesis de trabajo presentada, y teniendo en cuenta las limitaciones antes señaladas para un estudio de caso, se pudo ver cómo las variables de segmentación residencial (localización barrial) y segmentación socio-ocupacional (variable inserción socioocupacional) estructuran y articulan conformando tres tipos. La tipología inscripta en un plano factorial trazado por variables ordinales no sólo permite distinguir las componentes de cada uno de los tipos sino además “ordenar" los distintos tipos con base en criterios de urgencia. Resulta evidente detenerse en el tipo III que presenta la situación de mayor vulnerabilidad, pero es interesante detenerse también en el tipo II que es el más numeroso y que requiere otro enfoque de políticas públicas.

Las tipologías ofrecen la ventaja de poder dar cuenta de la multidimensionalidad de los fenómenos de segmentación. Como ocurre con los reclamos para una medición multidimensional de la pobreza, la situación de los hogares se debe a la concurrencia multidimensional de factores que podrían llevar a catalogar a un hogar de una determinada forma o de otra. Pero también es preciso observarlos en forma jerárquica y ordenada sobre el conjunto del plano.

Para los fines de este artículo, se pudo ver cómo funcionan como ejes articulantes la segmentación residencial y la socioocupacional, ambas como anverso y reverso de la se- 
gregación socioeconómica de la tradición de los estudios latinoamericanos.Y en el caso de esta localidad del GBA se hacen eco de la heterogeneidad estructural y de la relación entre pobreza e informalidad económica -una relación que tiene raíces históricas.

Pero además debe señalarse que ejercicios como éste tienen implicancias para el diseño de políticas sociales. Puede decirse, en un lenguaje coloquial, que hay dos definiciones de políticas sociales. Una, "corta", que remite a las acciones del Estado para los grupos más o menos vulnerables; otra, "larga", que engloba el conjunto de acciones del Estado que hacen al funcionamiento del capitalismo y el mercado de trabajo principalmente, así como el sostenimiento y acompañamiento de la reproducción de los hogares.

Una mirada sobre los tipos de nuestro análisis podría llevarnos a quedarnos con la definición corta al ver al conjunto de planes sociales y ayudas del tercer sector concentradas en el tipo III. Pero esta sería una lectura simplista, que pasaría por alto el momento histórico del relevamiento y la consolidación precisamente de estrategias destinadas a la atención de la emergencia (Halperín y Vinocur, 2004), que luego tuvieran mayor y mejor alcance con el desarrollo de los Programas de Transferencias Condicionadas de Ingresos (PTCI) (Rico, 2014). El desafio es despegarse de la mirada en la tipología solamente para mirar el conjunto del plano, en donde, todavía persisten los efectos segmentadores del mercado de trabajo (más evidente en este análisis) y de la especulación inmobiliaria (menos clara en este análisis). De la emergencia a los PTCI, se puede hablar de un pasaje de la asistencia a la redistribución (limitada) de ingresos. El próximo desafio es la transversalidad con el mercado de trabajo, para poder avanzar hacia una intervención más amplia. En este sentido, ejercicios como el realizado pueden ser de gran utilidad para el diseño de políticas de alcance multidimensional y transversal. 


\section{Anexo Metodológico}

\section{Variables-eje}

Para poder hacer ese agrupamiento, se trabaja con dos variables que son los factores ejes y que se espera ordenen al resto de las variables. La tabla a continuación presenta las variables y sus categorías:

\begin{tabular}{|l|l|}
\hline Variable & Categorías \\
\hline Localización barrial & Residente Zona Alta \\
\cline { 2 - 2 } & Recién llegado Zona Alta \\
\cline { 2 - 2 } & Residente Zona Baja \\
\cline { 2 - 2 } & Recién llegado Zona Baja \\
\hline Situación ocupacional del PSH & Empleadores y cuenta propia calificados \\
\cline { 2 - 2 } & Asalariados protegidos \\
\cline { 2 - 2 } & Dueños de establecimientos informales \\
\cline { 2 - 2 } & Asalariados informales \\
\cline { 2 - 2 } & $\begin{array}{l}\text { Cuenta propia eventuales, trabajo en hogares y } \\
\text { beneficiarios de programas }\end{array}$ \\
\hline
\end{tabular}

Respecto a la primera, va de suyo que se inscribe dentro de lo que se mencionaba al hablar de análisis territorial. Las categorías tienen en cuenta no sólo si la vivienda se ubica en la zona alta o baja, sino cuál es su antigüedad. Los que tienen una antigüedad mayor a 10 años en el barrio, son considerados residentes y a los demás se les asigna el término de "recién llegados". En este sentido, uno de los ejes que organizaría el perfil sería la zona en que se ubica la vivienda, suponiendo que tendría influencia la segregación socioresidencial.

El segundo eje tiene que ver con la calidad de la inserción socioocupacional del jefe/a teniendo en cuenta la categoría (empleador, cuentapropia, asalariado, trabajo en hogares) y la calidad (registrado o no registrado) ${ }^{13}$. También aquí se espera que esta variable condicione los ingresos y las estrategias del hogar. A su vez, ambas estarían actuando de forma complementaria dando lugar a una segmentación territorial y laboral: el residir en determinada zona condiciona el acceso a determinados tipos de empleo, y a su vez, el acceso a estos empleos determina las condiciones materiales de los hogares haciendo difícil la posibilidad de no residir en áreas segregadas. 
Pablo Molina Derteano

\section{Variables analíticas}

\begin{tabular}{|c|c|}
\hline \multicolumn{2}{|l|}{ Variables que se agruparon significativamente 1} \\
\hline Variable & Categorías \\
\hline \multirow[t]{4}{*}{ Calidad de materiales de la vivienda } & CALMAT I \\
\hline & CALMAT II \\
\hline & CALMAT III \\
\hline & CALMAT IV \\
\hline \multirow[t]{4}{*}{ Ayuda para el hogar } & Envía ayuda a otros hogares de familiares \\
\hline & Envía y recibe ayuda de familiares \\
\hline & Recibe ayuda de familiares \\
\hline & Recibe ayuda de terceros \\
\hline \multirow[t]{2}{*}{ Percepción de planes sociales } & Percibe \\
\hline & No percibe \\
\hline \multicolumn{2}{|l|}{ Variables que se agruparon parcialmente ${ }^{2}$} \\
\hline \multirow[t]{3}{*}{ Edad } & Hasta 29 años \\
\hline & 30 a 50 años \\
\hline & Mayores de 50 años \\
\hline \multirow[t]{4}{*}{ Regularidad dominial } & Propietario de vivienda y terreno \\
\hline & Propietario de vivienda \\
\hline & Inquilino \\
\hline & Otras \\
\hline \multirow[t]{3}{*}{ Nivel educativo del PSH } & Bajo: hasta primario incompleto \\
\hline & $\begin{array}{l}\text { Medio: primario completo hasta secundario } \\
\text { incompleto }\end{array}$ \\
\hline & Alto: Secundario completo y más \\
\hline \multicolumn{2}{|l|}{ Variables que no se agruparon ${ }^{3}$} \\
\hline \multirow[t]{2}{*}{ Sexo del PSH } & Varón \\
\hline & Mujer \\
\hline
\end{tabular}

1. Implica que dieron categorías a todas las tipologías

2. Implica que sus categorías no estuvieron presentes en todas las tipologías

3. Implica que las categorías se mantuvieron a distancias alejadas o no significativas de todas las tipologías 
${ }^{1}$ Vid. Carpio y Novacoksky (2000) y Carpio (2007, sobre el concepto de heterogeneidad estructural, vid Lavopa (2009) y Salvia (2011).

${ }^{2}$ Los datos primarios corresponden al proyecto FONCyT "Reproducción social de la nueva marginalidad urbana. Articulación de prácticas de acumulación en un sistema social dual y fragmentado" (PICT2005/ NRO.33737), bajo la dirección del Dr.Agustín Salvia. Se compone de una muestra probabilística de 530 casos, cuidando las cuotas de sexo y NBI y empleando los radios censales del censo de 2001, ya que el relevamiento se hizo en 2008. Se trató de una encuesta, que al igual que la EPH, posee dos unidades de análisis: hogares e individuos. La muestra que se analiza en este artículo se compone de 511 casos.

${ }^{3}$ Para un análisis del concepto y una aplicación metodológica de la investigación con tipos ideales, ver Molina Derteano (2014)

${ }^{4}$ Que abarca los municipios de Exaltación de la Cruz, Escobar, General Las Heras y General Rodríguez. Aun así en términos absolutos la población de esta área fue y es muy inferior a la del periurbano sur. ${ }^{5}$ Compuesto por los municipios de La Matanza, Merlo y Moreno.

${ }^{6}$ Tendencia que es aún más evidente en períodos intercensales anteriores, que aquí no son descritos por cuestiones de espacio. Para más información , ver el documento 17 de la Dirección Provincial de Estadística de Buenos Aires citado en bibliografia (Marzo de 2014)

${ }^{7}$ Compuesto por los municipios de Avellaneda, Lanús, Lomas de Zamora y Quilmes.

${ }^{8}$ Compuesto por los municipios de General San Martín, Morón, San Isidro, Tres de Febrero y Vicente

López.

${ }^{9}$ Como lo indican los datos en el cuadro 1, la zona de mayor peso poblacional en 1947 era el conurbano sur. Si tomamos la población de esa zona como 100 y la mantenemos en los sucesivos períodos intercensales, podemos ver la magnitud de los cambios. En 1947, la población de la zona periurbana sur era el 26\% de la de la zona del conurbano sur; en 2001 , era $80 \%$ y en 2010 el $88 \%$.

${ }^{10} \mathrm{Si}$ se aplicara un criterio empírico radical, se debería cruzar todas las variables entre sí y ver cuál es el par con mayor inercia. Pero se corre el riesgo de correr el eje del análisis. Así se daba el caso de que la inercia entre máximo nivel educativo del jefe de hogar y su inserción ocupacional era mayor que otras, pero correría el eje de un análisis territorial hacia uno de capital humano o mercado de trabajo.

${ }^{11}$ Adicionalmente, fue la asociación más fuerte que involucrara la variable localización barrial

${ }^{12}$ La escala CALMAT, referida a la calidad de los materiales de construcción, ha sido tomada de Chávez Molina y Gutiérrez (2010).

${ }^{13}$ Esta calidad es medida a través de la declaración de hacer o tener descuentos jubilatorios en la ocupación actual. 


\section{Referencias bibliográficas}

ACUMAR (2013) Población con Necesidades Básicas Insatisfechas, mimeo

Arzaluz Solano, Socorro (2005) "La utilización del estudio de caso en análisis local" en Región y Sociedad Vol XVII, N 32:107-144.

AAVV [Gobierno municipal de Almirante Brown - Facultad de Arquitectura, Diseño y Urbanismo (UBA) y Fundación Metropolitana] (2010). Programa de fortalecimiento Institucional para el Municipio de Almirante Brown. Diagnóstico urbano de Almirante Brown.Etapa 1

Barbosa, Eva (2001). Urban spatial segregation and social differentiation: foundation for a typological analysis. Lincoln Institute of Land Policy, conference paper.

Beccaria Luis y Groissman, Fernando (2008) "Informalidad y pobreza en Argentina”. Investigación Económica, (UNAM) LXVII (266)135-169.

Beccaria Luis y Groissman, Fernando (1978) "Una contribución al estudio de la movilidad social en la Argentina. Análisis de los resultados de una encuesta para el Gran Buenos Aires”, en Desarrollo Económico N 68, Vol 17: 234-287.

Bourdieu, Pierre (2002). "Efectos de lugar”. En P. Bourdieu (Ed.), La miseria del mundo. Buenos Aires: FCE.

Carpio Jorge, Klein, Emilio y Novakovsky, Irene (2000) "Introducción” en Carpio, J., Klein, E. y Novakovsky, I. (Comp.), Informalidad y Exclusión Social, Buenos Aires: Fondo de Cultura Económica, SIEMPRO, OIT.pp 11-24

Chávez Molina Eduardo y Sacco, Nicolás (2014) Estructura de clases basadas en la heterogeneidad estructural. Su evolución distributiva en los últimos 20 años. En III Jornadas nacionales sobre estudios regionales y mercados de trabajo. 3 y 4 de septiembre de 2014 , San Salvador de Jujuy.

Chávez Molina Eduardo y Sacco, Nicolás (2013) Movilidad social en contexto de heterogeneidad estructural. Ponencia en el II Seminario Internacional de Desigualdad y Movilidad social en América Latina, Mendoza, 28 al 30 de junio de 2013.

Chávez Molina Eduardo y Sacco, Nicolás (2010) La construcción social en el mercado informal. Los feriantes de Francisco Solano. Buenos Aires: NuevaTrilce. 
Chávez Molina Eduardo y Sacco, Nicolás y Gutiérrez, Pablo (2010) Las huellas de la intervención social. Buenos Aires:Trilce.

Chávez Molina Eduardo y Sacco, Nicolás (2005) Informe sobre las condiciones de vida $y$ vivienda en Ministro Rivadavia, SEDECA

Clemente, Adriana (2014) "Sobre la pobreza como categoría de análisis e intervención", en Clemente, Adriana (comp.) Territorios Urbanos y Pobreza Persistente. Buenos Aires: Espacio Editorial:23-62

Dirección Provincial de Estadística (2014) La población de la provincia de Buenos Aires. Aspectos salientes de su crecimiento intercensal. Período 1947-2010, Serie documentos demográficos No 17, La Plata: Gobierno de la Provincia de Buenos Aires.

Dirección Provincial de Estadística (2012) Censo 2010. Provincia de Buenos Aires. Resultados definitivos por partido. La Plata: Gobierno de la Provincia de Buenos Aires.

Echeverría, Andrea (2014) "El barrio como parte de la solución y como problema" en Clemente, Adriana (comp.) Territorios Urbanos y Pobreza Persistente. Buenos Aires: Espacio Editorial. pp169-184

Flyvberg, Bent (2004) "Cinco malentendidos acerca de la investigación mediante los estudios de caso", en REIS 106/04:33-62.

Galín, Pedro (2000) "Formas de protección de los trabajadores precarios". Derecho del Trabajo, No 9, Buenos Aires.

Germani, Gino (1967) "La ciudad como mecanismo integrador", en Revista Mexicana de Sociología Vol 29, número 3:387-406.

Gómez Rojas Gabriela y Riveiro, Manuel (2014) “El análisis de conglomerados en la construcción de tipos. El caso de la clasificación de parejas según la división de trabajo doméstico". En Entramados y Perspectivas Vol 4, No 4:93-114

Halperin, Leopoldo y Vinocur, Pablo (2004) "Pobreza y políticas sociales en Argentina de los años noventa". Santiago de Chile: CEPAL, Serie Politicas Sociales 85.

INDEC (2005) ¿Qué es el GBA?, Buenos Aires:INDEC.

Kessler, Gabriel (2013) Controversias sobre la desigualdad. Argentina 2003-2013. Buenos Aires: FCE. 
Pablo Molina Derteano

Lavopa, Alejandro (2009) Heterogeneidad estructural productiva argentina: impacto en el mercado laboral durante el período 1991-2003,Documentos de trabajo 9, CEPED, Buenos Aires.

López Roldan, Pedro; Artiles, Martín y Molina Oscar (2013) “Movilidad ascendente de la inmigración en España ¿asimilación o segmentación ocupacional?” En Chávez Molina, Eduardo (comp.) Desigualdad y movilidad en el mundo contemporáneo. Buenos Aires:Imago Mundi, 189-218.

López Roldan, Pedro; Artiles, Martín y Molina Oscar (1996) "La construcción de una tipología de segmentación del mercado de trabajo”. En papers 48: 9-29.

Molina Derteano, Pablo (2014) Herederos de una venganza. Un ejercicio con tipos ideales para el análisis de juventudes de clases medias altas y bajas. En I congreso Internacional de Metodología Cualitativa, Córdoba, 1 al 3 de octubre de 2014.

Molina Derteano, Pablo (2013) "Primeras aproximaciones para el estudio de los procesos de estratificación en los aglomerados urbanos en Argentina”. En Revista Confluencia, $\mathrm{N}^{\circ} 13: 55-82$.

Molina Derteano, Pablo (2007) "Sueños del eterno retorno de la sociedad salarial para los jóvenes asalariados precarios en condiciones de segmentación territorial”, en Salvia A. y Chávez Molina E. (comp.) Sombras de una marginalidad fragmentada. Aproximaciones a la metamorfosis de los sectores populares de la Argentina, Buenos Aires: Miño y Dávila, 241-268.

Ministerio de Desarrollo Social (2011). Políticas Sociales del Bicentenario. Un Modelo Nacional y Popular. Tomo I. Buenos Aires.

Palma Arce, Carolina y Soldano Daniela (2010) “Capital espacial y movilidad cotidiana en la Región Metropolitana de Buenos Aires. Una propuesta analítica y empírica". En Roffman, A. (comp.) Sociedad y territorio en el conurbano bonaerense, Buenos Aires: Universidad Nacional de General Sarmiento, 103-134.

Piore, Michel (1983) Paro e inflación. Madrid:Alianza.

Portes, Alejandro (1989) En torno a la informalidad: ensayos sobre teoría y medición de la economía no regulada. México: Miguel Porrúa.

Quijano, Anibal (2012) "Polo marginal y mano de obra marginal”, En Quijano Anibal. Cuestiones y horizontes. Antología esencial. Buenos Aires:CLACSO. 
Rico, Nieves (2014) Transferencia de ingresos para la erradicación de la pobreza. Dos décadas de experiencias en los países de la Unión de Naciones Suramericanas (UNASUR), CEPALUNASUR.

Rodríguez Vignoli, Jorge (2001) “Segregación residencial socioeconómica: ¿Qué es? ¿Cómo se mide?, ¿qué está pasando? ¿Importa?," Santiago de Chile: CEPAL, Serie Población y desarrollo $\mathrm{N}^{\circ} 16$.

Roffman, Adriana (2010) “Introducción”, en Roffman,A. (comp.) Sociedad y territorio en el conurbano bonaerense, Buenos Aires: Universidad Nacional de General Sarmiento, 9-24.

Salvia, Agustín (2011) La trampa neoliberal. Buenos Aires: Eudeba.

Saraví, Gonzalo (2008) "Mundos aislados: segregación urbana y desigualdad en la ciudad de México". EURE (Santiago) 2008, vol.34, n.103 pp. 93-110[onli$n e$. Disponible en: $<$ http://www.scielo.cl/scielo.php?script=sci_arttext\&pid=S0250$71612008000300005 \& \operatorname{lng}=$ es\&nrm $=$ iso $>$. ISSN 0250-7161.

http://dx.doi.org/10.4067/S0250-71612008000300005.Accesado 27/01/2015

Saraví, Gonzalo (2004) “Segregación urbana y espacio público: los jóvenes en enclaves de pobreza estructural”. Revista de la CEPAL 83:33-48.

Schneider, Sergio y PeyréTartuga, Iván (2006). “Territorio y enfoque territorial: de las referencias cognitivas a los aportes aplicados al análisis de los procesos sociales rurales”. En Manzanal, M.; Neiman, G. y Lattuada M. (orgs.) Desarrollo rural. Organizaciones, instituciones y territorios. Buenos Aires: Ciccus, 71-102.

SIEMPRO (2007): Evaluación de medio término del Plan Manos a la Obra. Buenos Aires: Jefatura de Gabinete de Ministros.

Sosa Velazquez, Mario (2012) ¿Cómo entender el territorio? Guatemala: Cara Parens.

Tokman, Victor (2002) "La informalidad en los años noventa. Situación y perspectivas”, en Carpio J. y Novacovsky, I. (comps.) De igual a igual. El desafío del Estado ante los nuevos problemas sociales. Buenos Aires: SIEMPRO,103-138

Tokman, Victor y Souza, Paulo (1978) “Distribución del ingreso, pobreza y empleo en áreas urbanas”. El Trimestre Económico,Vol. 45, No. 179(3):737-766. 